\title{
Reconfigurable Antenna Based DoA Estimation and Localization in Cognitive Radios: Low Complexity Algorithms and Practical Measurements
}

\author{
Aki Hakkarainen*, Janis Werner*, Nikhil Gulati ${ }^{\dagger}$, Damiano Patron $^{\dagger}$, Doug Pfeil ${ }^{\dagger}$, \\ Henna Paaso ${ }^{\ddagger}$, Aarne Mämmelä ${ }^{\ddagger}$, Kapil Dandekar ${ }^{\dagger}$ and Mikko Valkama* \\ *Department of Electronics and Communications Engineering, Tampere University of Technology, Tampere, Finland \\ Emails: \{aki.hakkarainen, janis.werner, mikko.e.valkama\}@tut.fi \\ ${ }^{\dagger}$ Department of Electrical and Computer Engineering, Drexel University, Philadelphia, PA 19104, USA \\ Emails: \{ng54, dp578, dsp36, krd26\}@ drexel.edu \\ $\ddagger$ VTT Technical Research Centre of Finland, Oulu, Finland \\ Emails: \{henna.paaso, aarne.mammela\}@vtt.fi
}

\begin{abstract}
This paper addresses low-complexity algorithms and evaluates the practical performance of low-complexity primary user (PU) direction of arrival (DoA) estimation and PU localization with real world indoor measurement data. More specifically, we use a type of reconfigurable antenna known as leaky-wave antennas to sense the spatial distribution of the $P U$ signal power. By deploying a very low-complexity algorithm, called MaxE, the secondary user (SU) sensors are then able to estimate their respective PU DoAs. Finally, a central fusion center combines the DoAs into a PU location estimate. The results of the practical measurements reveal that it is possible to implement a localization system with very low complexity and fairly good PU location capabilities in a cognitive radio network. Such PU localization capabilities can then be used, e.g. for enhanced PU interference management.
\end{abstract}

Index Terms-Cognitive radio, direction-of-arrival estimation, leaky-wave antennas, localization, low complexity, measurements, reconfigurable antennas, Stansfield algorithm

\section{INTRODUCTION}

Primary user (PU) location information is required to enable several key capabilities in cognitive radio (CR) networks, such as improved spatial-temporal sensing, intelligent locationaware routing and spectrum policy enforcement [1]. As the PUs cannot be assumed to cooperate with the CR network, their locations have to be estimated based on their transmissions alone. Towards that end, the cooperation of multiple secondary users (SUs) is essential in order to guarantee accurate localization of PUs over the whole coverage area.

This work is supported by the Finnish Funding Agency for Technology and Innovation (Tekes) under the project "Reconfigurable Antenna-Based Enhancement of Dynamic Spectrum Access Algorithms", the Industrial Research Fund of Tampere University of Technology (Tuula and Yrjö Neuvo Fund), the Academy of Finland under the project 251138 "Digitally-Enhanced RF for Cognitive Radio Devices", the Doctoral Programme of the President of Tampere University of Technology, and the Foundation of Nokia Corporation.

The work is also supported by National Science Foundation (NSF) under award number 1147838. We would also like to thank Adant Technologies Inc. for the antenna prototypes.
Location estimation of a non-cooperative transmitter can be classified into three categories, namely received-signal strength (RSS), time-difference-of-arrival (TDoA) and direction-ofarrival (DoA) based methods [2]. However, TDoA requires perfect synchronization among the localizing nodes, which is hard to realize in a CR network. Therefore, prior research has focused mainly on RSS [3], [4], DoA [5] or hybrid RSS/DoAbased PU localization [6], [7].

A particular problem of DoA-based localization in CR networks has been the size and cost of the required devices, as well as the high complexity of the assosicated DoA estimation algorithms [8]. Many classical DoA estimators, such as the popular multiple signal classification (MUSIC) algorithm [9] require large antenna arrays and result in a high processing complexity that may be prohibitive in a CR network, where the SUs contribute to the localization. We have therefore recently proposed to use reconfigurable antennas for PU DoA estimation in CR networks [8], [10]. While we have demonstrated that reconfigurable antennas can be used for DoA estimation of a cooperating transmitter in realistic conditions [11], we are yet to show the same for DoA estimation of a non-cooperating transmitter. A promising candiate for DoA estimation of a non-cooperating PU is the MaxE algorithm [8]. Since its computational complexity is exceptionally low, it can be employed within the SUs while causing only a small localization overhead. In this paper, we therefore present practical measurement results of MaxE based DoA estimation as well as successive localization based on the Stansfield fusion algorithm [12]. As the original MaxE algorithm was conceived for antennas whose radiation patterns are independent of the direction that the antenna is steered to, we propose in this paper an equalization that compensates for the antenna's varying gains in different directions.

This paper is organized as follows. A detailed overview of the localization system is presented in Section II while the practical measurement setup and the used reconfigurable antennas are discussed in Section III. Section IV evaluates 


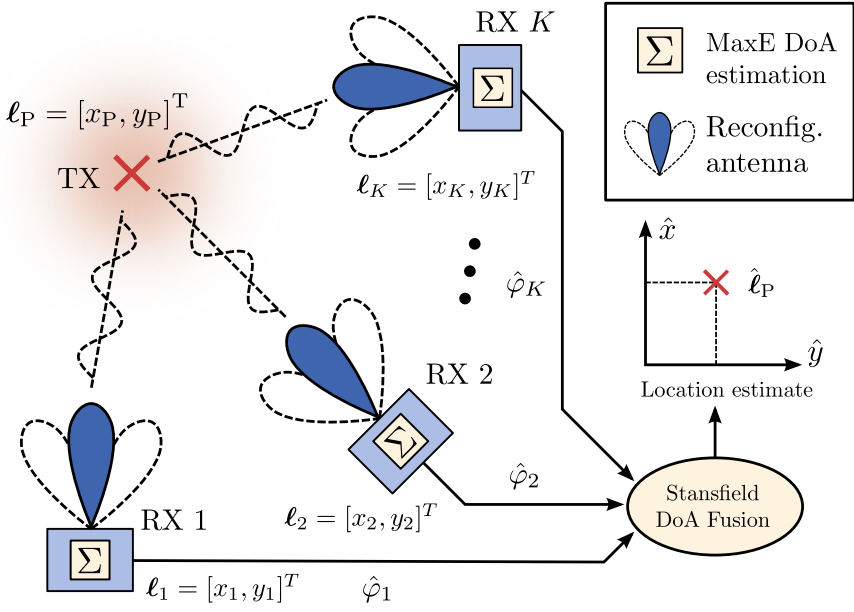

Fig. 1: Localization system: The non-cooperative PU can be abstracted as a TX, while each of the cooperating SUs can be modeled as RXs. DoA estimation takes place within every RX using the MaxE algorithm. The estimated DoAs are then communicated to a central fusion center that estimates the TX location through the Stansfield algorithm.

the performance of the localization system using the obtained measurements. The paper is concluded in Section V, with an additional outlook on our future work.

\section{OVERVIEW OF THE LOCALIZATION SYSTEM}

Fig. 1 shows an illustration of the localization system under consideration. As we are dealing with transmissionbased PU localization, we can model the PU as a simple transmitter (TX) and the localizing SUs as receivers (RXs) that are equipped with reconfigurable antennas. Each $\mathrm{RX}$ $k, k=1 \ldots K$, estimates the respective TX signal DoA $\varphi_{k}$ using MaxE algorithm (Section II-A) and communicates the DoA estimate as well as its known location $\ell_{k}=\left[x_{k}, y_{k}\right]^{T}$ to a fusion center where the TX location, $\ell_{\mathrm{P}}=\left[x_{\mathrm{P}}, y_{\mathrm{P}}\right]^{T}$, is estimated as $\hat{\ell}_{\mathrm{P}}$ using the Stansfield algorithm (Section II-B).

\section{A. DoA Estimation}

In theory, a reconfigurable antenna can sweep through the angular range continuously. However, it cannot be assumed that the TX is transmitting continuously. Moreover, DoA estimation should require as little time as possible. Therefore, we assume that $\mathrm{RX} k, k=1 \ldots K$ directs its reconfigurable antenna in $M$ sectors with main beam orientations $\vartheta_{k, 1}, \ldots, \vartheta_{k, M}$ and observes a power measurement $\epsilon_{k, m}$ in every sector $m$. Using these measurements, the MaxE algorithm [8] then estimates the DoA as

$$
\hat{\varphi}_{k}=\left\{\vartheta_{k, m} \mid m=\arg \max _{m} \epsilon_{k, m}\right\} \text {. }
$$

However, (1) assumes a constant attenuation in every sector. In practice this may not be the case due to variable directional gain as can also be seen from the gain measurement results shown in Table I. Therefore, in this paper we propose to equalize the powers prior to MaxE DoA estimation, resulting in

$$
\tilde{\varphi}_{k}=\left\{\vartheta_{k, m} \mid m=\arg \max _{m} \frac{\epsilon_{k, m}}{\alpha_{m}}\right\} .
$$

Naturally, the equalization coefficients, $\alpha_{m}$, should be chosen as the average attenuation that sector $m$ is causing to an incoming signal. Without any additional information, we have to assume a uniformly distributed DoA and obtain

$$
\alpha_{m}=\frac{1}{360} \int_{-180}^{180} p_{m}(\varphi) d \varphi
$$

where $p_{m}(\varphi)$ denotes the attenuation of sector $m$. In practice, $p_{m}(\varphi)$ is not available as a closed-form function. Instead, the equalization coefficients may be obtained by measuring $p_{m}(\varphi)$ in $N$ discrete steps $p_{m}(n \beta), n=1 \ldots N$ with step size $\beta$ and replacing the integral in (3) by a sum.

\section{B. Localization}

The Stansfield algorithm [12] estimates the TX location as

$$
\hat{\ell}_{P}=\left(\mathbf{A}^{\mathrm{T}} \mathbf{W} \mathbf{A}\right)^{-1} \mathbf{A}^{\mathrm{T}} \mathbf{W} \mathbf{b}
$$

with

$$
\begin{aligned}
\mathbf{A} & =\left(\begin{array}{cc}
\sin \left(\hat{\varphi}_{1}\right) & -\cos \left(\hat{\varphi}_{1}\right) \\
\vdots & \vdots \\
\sin \left(\hat{\varphi}_{K}\right) & -\cos \left(\hat{\varphi}_{K}\right)
\end{array}\right) \\
\mathbf{b} & =\left(\begin{array}{c}
x_{1} \sin \left(\hat{\varphi}_{1}\right)-y_{1} \cos \left(\hat{\varphi}_{1}\right) \\
\vdots \\
x_{K} \sin \left(\hat{\varphi}_{K}\right)-y_{K} \cos \left(\hat{\varphi}_{K}\right)
\end{array}\right)
\end{aligned}
$$

where $\mathbf{W}=\operatorname{diag}\left(w_{1}, \ldots, w_{K}\right)$ is a weighting matrix that can be set to [8]

$$
w_{k}=\max _{m} \epsilon_{k, m}
$$

We note that the computational complexity of Stansfield location estimation is large compared to MaxE DoA estimation. However, since only the DoA is estimated within the RXs while the location estimate is obtained in a dedicated fusion center, this is exactly the kind of localization system suitable for a CR network.

\section{Measurement Setup and Reconfigurable ANTENNA STRUCTURE}

\section{A. Measurement Procedure and Equipment}

In order to evaluate the practical perfomance of the DoA estimation and localization algorithms, we arranged an extensive measurement campaign in a large lobby area which is depicted in Fig. 2. There were several active WiFi hotspots nearby, causing some in-band interference. In addition, the passers-by generated spatial and temporal variations in the measurement results.

We used three TX and six RX locations which are illustrated in Fig. 3. These locations were selected for testing the 


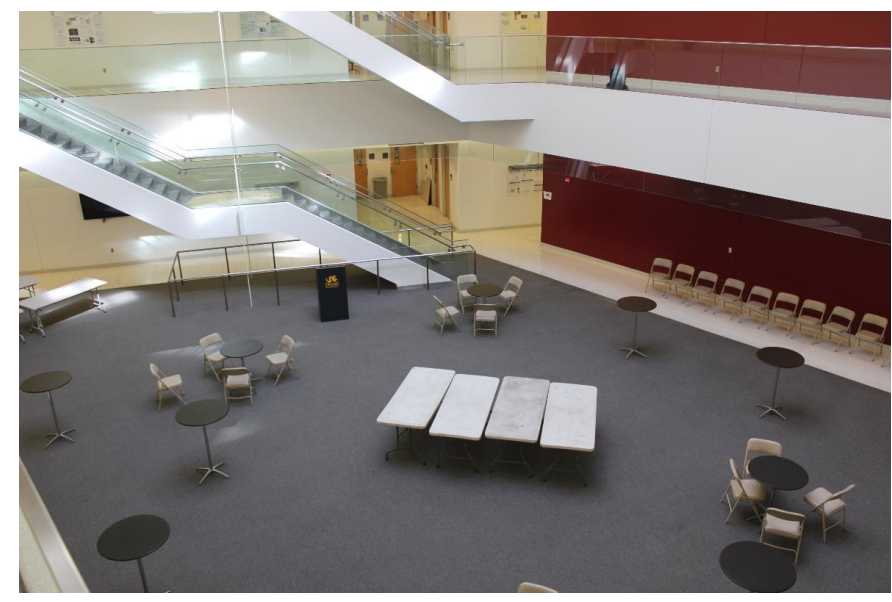

Fig. 2: Measurements were done in this spacious lobby area in the Drexel University premises. During the measurements, all tables and chairs were placed on the sides of the lobby.

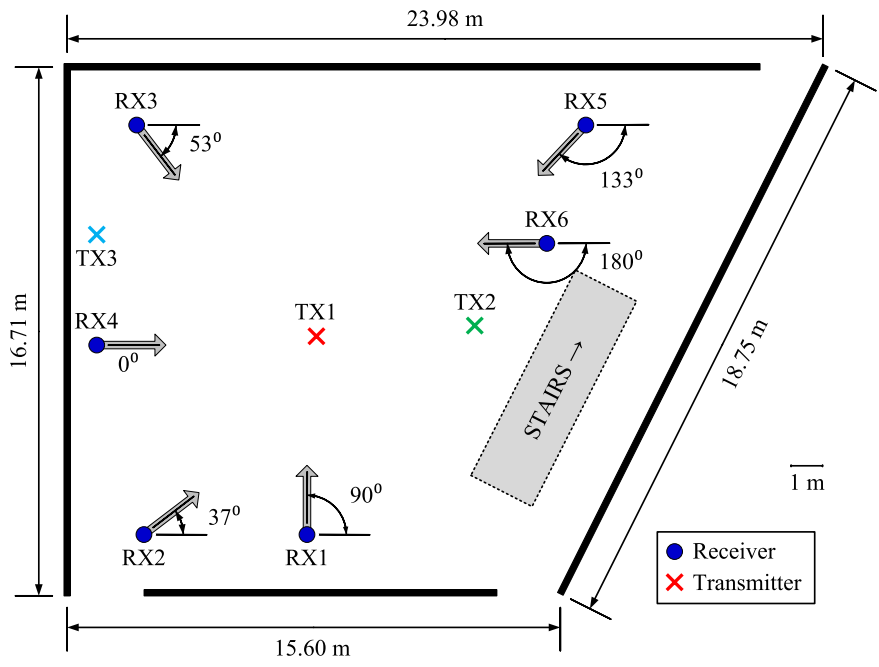

Fig. 3: Layout of the measurement area and the locations of the TXs and RXs. The grey arrows indicate the orientations of the antenna broadsides.

performance in different challenging estimation scenarios. The $\mathrm{RX}$ antennas were oriented in such way that they can hear the TXs within their directivity ranges in most of the cases.

Each transceiver consisted of a field programmable gate array (FPGA) based software defined radio platform, called Wireless Open-Access Research Platform (WARP) v3 [13]. Each WARP board was connected to its own antenna(s) and to a centralized controlling system. The TXs were equipped with two omni-directional antennas whereas each RX had a single leaky-wave antenna (LWA) with two antenna ports. The concept of the LWA is described in more detail in Section III-B.

The system was operating with $20 \mathrm{MHz}$ channel bandwidth and the carrier frequency was $2.462 \mathrm{GHz}$. This combination is heavily overlapping with the WiFi channel no. 11 which

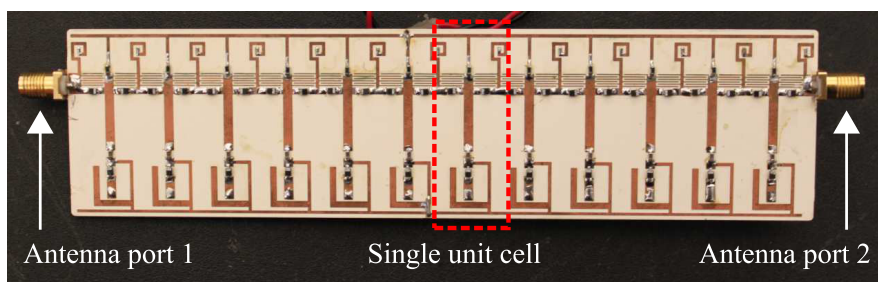

Fig. 4: An illustration of LWAs used in the measurements. The LWA consists of two antenna ports and 12 unit cells in row. The physical size of the antenna is $156 \mathrm{~mm} \times 38 \mathrm{~mm}$.

was measured to have active traffic during the measurements. We used orthogonal frequency division multiplexing (OFDM) waveforms carrying symbols with binary phase shift keying (BPSK) modulation. The total amount of subcarriers was 64 of which 48 were used for carrying data. Only one of the transmission links, i.e. TX-RX antenna pair, was active at a time and thus we needed to ensure a fairness between different transmission links by transmitting the same data over all links. Note that realistically all RXs could receive the same transmitted data, propagated through different channels though, and therefore the measurement arrangement matches the real world scenario well. For testing each link, we transmitted in total 300 packets each containing 5420 BPSK symbols. The TX power was set to $+15 \mathrm{dBm}$ and since our algorithms rely on the received signal powers, automatic gain controls (RF and baseband) were deactivated in the RXs and the gains were set to constant values.

All measurement results were saved for post-processing with the algorithms discussed in Section II. For each antenna sector, we calculated the received signal power from the baseband signal snapshots by averaging over the powers of all received packets observed through the considered antenna sector.

\section{B. Composite Right/Left-Handed Leaky-Wave Antenna}

LWAs belong to the class of traveling-wave antennas [14]. In contrast to conventional resonating-wave antennas, LWAs leak out energy progressively as the wave travels along the microstrip structure. The main beam of the LWA is normal to the plane of the antenna and can be in general steered by changing the electrical properties of the radiating elements.

The reconfigurable composite right/left-handed LWA strucure consists of a cascade of metamaterial unit cells [15]. In the measurements, we used LWAs which were tuned to operate within the entire $2.4 \mathrm{GHz} \mathrm{WiFi}$ band. A photo of the antenna can be found in Fig. 4. The physical size of the antenna is $156 \mathrm{~mm} \times 38 \mathrm{~mm}$ and the antenna consist of 12 unit cells for obtaining good directivity and a small form factor simultaneously. Each unit cell is populated with two varactor diodes in series and one in shunt configuration. The main beam of the antenna can be steered from the broadside direction backward and forward by changing two DC control voltages which in turn change the behavior of the travelingwave. Due to the practically symmetric antenna structure, 


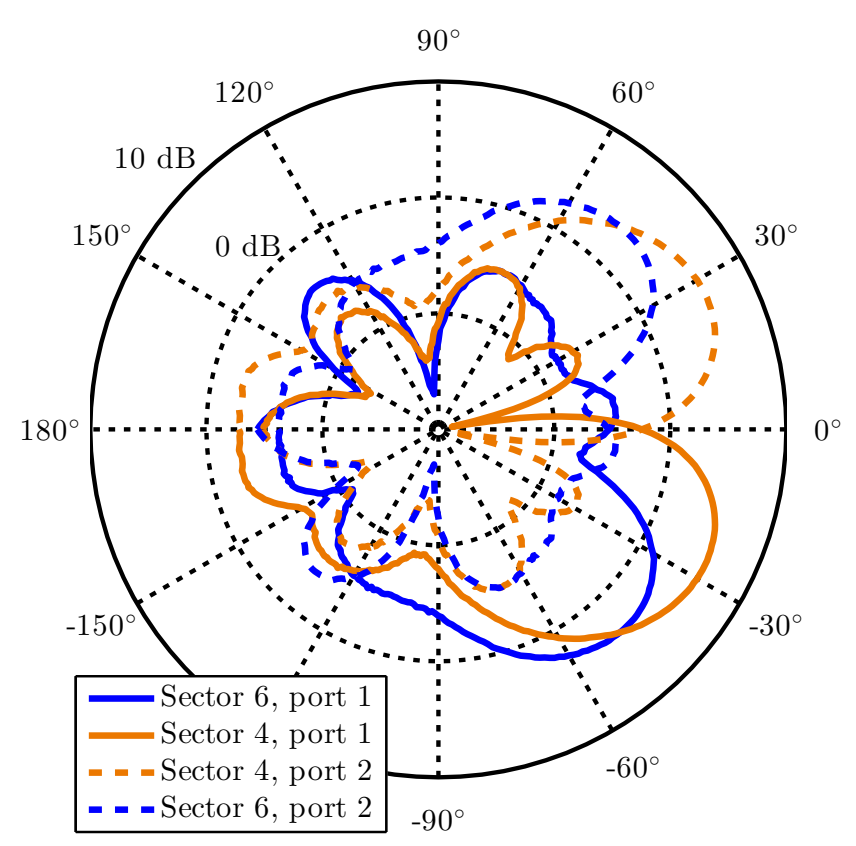

Fig. 5: Measured radiation patterns of four different antenna sectors. Note that port 1 and port 2 have symmetric radiation properties.

TABLE I: Measured antenna main beam orientations and gains

\begin{tabular}{c|c|c} 
Sector & Main beam orientation & Main beam gain \\
\hline \hline 1 & $0^{\circ}$ & $5.0 \mathrm{~dB}$ \\
2 & $\pm 8^{\circ}$ & $5.1 \mathrm{~dB}$ \\
3 & $\pm 18^{\circ}$ & $5.6 \mathrm{~dB}$ \\
4 & $\pm 28^{\circ}$ & $5.8 \mathrm{~dB}$ \\
5 & $\pm 39^{\circ}$ & $4.9 \mathrm{~dB}$ \\
6 & $\pm 47^{\circ}$ & $3.5 \mathrm{~dB}$
\end{tabular}

antenna ports have symmetric radiation properties with respect to the broadside direction. This beam symmetry is depicted in Fig. 5, which illustrates four measured radiation patterns (two for each antenna ports) for different control voltages. By properly setting the control voltages, the used LWAs are able to sweep their main beam orientations approximately from $-50^{\circ}$ to $+50^{\circ}$. As a trade-off between estimation time and estimation accuracy, we have used 6 control voltage pairs for a total of 12 radiation sectors. The measured main beam directions and the corresponding gains are shown in Table I.

\section{Performance Evaluation and Analysis}

\section{A. DoA Estimation}

The measurement data was post-processed by using the low-complexity MaxE algorithm for obtaining DoA estimates for each TX. Table IIa shows the resulting errors of DoA estimation when using the basic MaxE algorithm, i.e. powers from different antenna sectors are not equalized. Based on the results, there are clear differences in the estimation accuracy obtained by different RXs. As expected, RX1 performs very well and reaches the lowest root-mean-square error (RMSE)
TABLE II: Absolute error of DoA estimation

(a) No power equalization, equation (1)

\begin{tabular}{c|c|c|c|c|c|c|c} 
& $\mathrm{RX} 1$ & $\mathrm{RX} 2$ & $\mathrm{RX} 3$ & $\mathrm{RX} 4$ & $\mathrm{RX} 5$ & $\mathrm{RX} 6$ & $\mathrm{RMSE}$ \\
\hline \hline $\mathrm{TX} 1$ & $2.7^{\circ}$ & $29.7^{\circ}$ & $5.1^{\circ}$ & $20.3^{\circ}$ & $19.5^{\circ}$ & $6.1^{\circ}$ & $17.0^{\circ}$ \\
$\mathrm{TX} 2$ & $0.2^{\circ}$ & $22.9^{\circ}$ & $21.8^{\circ}$ & $5.1^{\circ}$ & $6.4^{\circ}$ & $9.8^{\circ}$ & $13.9^{\circ}$ \\
$\mathrm{TX} 3$ & $12.0^{\circ}$ & $22.6^{\circ}$ & $10.5^{\circ}$ & $43.0^{\circ}$ & $13.0^{\circ}$ & $26.9^{\circ}$ & $24.2^{\circ}$ \\
\hline $\mathrm{RMSE}$ & $7.1^{\circ}$ & $25.3^{\circ}$ & $14.3^{\circ}$ & $27.6^{\circ}$ & $14.0^{\circ}$ & $16.9^{\circ}$ &
\end{tabular}

(b) Power equalization, equation (2)

\begin{tabular}{c|c|c|c|c|c|c|c} 
& $\mathrm{RX} 1$ & $\mathrm{RX} 2$ & $\mathrm{RX} 3$ & $\mathrm{RX} 4$ & $\mathrm{RX} 5$ & $\mathrm{RX} 6$ & $\mathrm{RMSE}$ \\
\hline \hline $\mathrm{TX} 1$ & $2.7^{\circ}$ & $29.7^{\circ}$ & $5.1^{\circ}$ & $10.3^{\circ}$ & $0.5^{\circ}$ & $3.9^{\circ}$ & $13.1^{\circ}$ \\
$\mathrm{TX} 2$ & $8.2^{\circ}$ & $22.9^{\circ}$ & $21.8^{\circ}$ & $5.1^{\circ}$ & $6.4^{\circ}$ & $1.8^{\circ}$ & $13.8^{\circ}$ \\
$\mathrm{TX} 3$ & $12.0^{\circ}$ & $14.6^{\circ}$ & $10.5^{\circ}$ & $43.0^{\circ}$ & $13.0^{\circ}$ & $26.9^{\circ}$ & $23.1^{\circ}$ \\
\hline RMSE & $8.5^{\circ}$ & $23.3^{\circ}$ & $14.3^{\circ}$ & $25.7^{\circ}$ & $8.4^{\circ}$ & $15.7^{\circ}$ &
\end{tabular}

due to the good coverage and interference-free location. In contrast to that, RX2 and RX4 yield relatively high RMSEs. The results of RX2 are most probably affected by harmful reflections from the stairs (made of metal, concrete and glass) whereas the high RMSE of RX4 follows from the fact that TX3 is actually out of the angular range of RX4. When the results are analyzed row-wise, we see that TX3 is clearly the most difficult to be estimated, as expected due to its somewhat isolated location. In addition, TX1 and TX2 are easier since they are located on very central places and thus can be well heard by all RXs.

Similar DoA estimation results for the MaxE with the proposed power equalization are given in Table IIb. Now the known differences between the sector gains are included in the processing and that results naturally in performance improvements. When comparing the results in different columns to Table IIa, we notice that power equalization decreases the RMSE in four cases, increases the RMSE in the RX1 case and does not affect the RMSE in the RX3 case. Actually, the performance is degraded only with one transmission link, namely TX2-RX1. A clear reason can not be found from the measurement setup and thus this one-time increase of the error is most likely caused by some transient error source, e.g. measurement equipment malfunction (antenna control voltage failure) or external interference (passers-by or WiFi traffic). When analyzing the results row-wise, the meaning of a single and possibly faulty observation is smaller due to the higher number of observations. This is actually yielding better RMSE for all TXs. All in all, it is clear that DoAs can be estimated in practice with a fairly good accuracy even with the very lowcomplexity MaxE algorithm and fairly coarse sectorization capabilities of the antennas.

\section{B. Localization}

After the DoA estimates from different RXs are sent to the fusion center, the location estimates of the TXs can be obtained by using the well-known Stansfield fusion algorithm. The localization errors are presented in Table III where the localization is based on the DoA estimates both without 
TABLE III: Error in localization

(a) No power equalization, equation (1)

\begin{tabular}{c|c|c|c||c} 
& TX1 & TX2 & TX3 & RMSE \\
\hline \hline Absolute error & $0.7 \mathrm{~m}$ & $4.4 \mathrm{~m}$ & $2.3 \mathrm{~m}$ & $2.9 \mathrm{~m}$
\end{tabular}

(b) Power equalization, equation (2)

\begin{tabular}{c|c|c|c||c} 
& TX1 & TX2 & TX3 & RMSE \\
\hline \hline Absolute error & $0.8 \mathrm{~m}$ & $4.5 \mathrm{~m}$ & $1.8 \mathrm{~m}$ & $2.8 \mathrm{~m}$
\end{tabular}

and with the power equalization. When comparing these two methods, we see that TX1 and TX2 location estimates have very similar accuracy with both DoA estimation methods. However, the power equalized MaxE outperforms the other version clearly when estimating TX3. Based on the results, TX1 can be localized very precisely as the location error is around $80 \mathrm{~cm}$. In contrast, TX2 can not be localized accurately. This is a bit surprising since the DoA RMSEs of TX1 and TX2 are very close to each others. We believe that the localization problem in the TX2 case is caused by accumulative DoA errors which in turn may follow from the rich or strong multipath environment. The location error of TX3 is a very good result when taking into account the original DoA estimates with the relatively high error levels. The total location RMSE with the proposed power equalization is $2.8 \mathrm{~m}$ which corresponds to a circle with an area of $7.5 \%$ of the whole measurement area. The measurement area and the obtained location estimates from the DoA estimates with power equalization are illustrated in Fig. 6. Altogether, Table III and Fig. 6 show that low-complexity algorithms can provide good abilities for localizing non-cooperative TXs not only in theory but also in practice. The location estimation accuracy could be even further improved in an easy and scalable manner if the number of RXs and thus the number of observations was higher.

\section{CONCLUSions AND Future Work}

In this paper we demonstrated that reconfigurable antennas in combination with very low-complexity algorithms can be used to localize a non-cooperative PU in a CR system. In particular, we used indoor measurement data that was obtained in realistic challenging conditions with rich scattering and cochannel interference to evaluate the achievable performance of MaxE DoA estimation with successive collaborative localization using the Stansfield algorithm. Our results reveal that even with a low number of collaborating SUs and despite the challenging environment that included fading, multipath propagation and interference from active WiFi systems, the localization system was able to localize the PUs with fairly good accuracy.

In the future, we will consider reconfigurable antenna-based localization using the simplified least squares (SLS) DoA estimator [8]. SLS has been shown to outperform the MaxE algorithm in theory [8], [10], [16], at the cost of being slightly

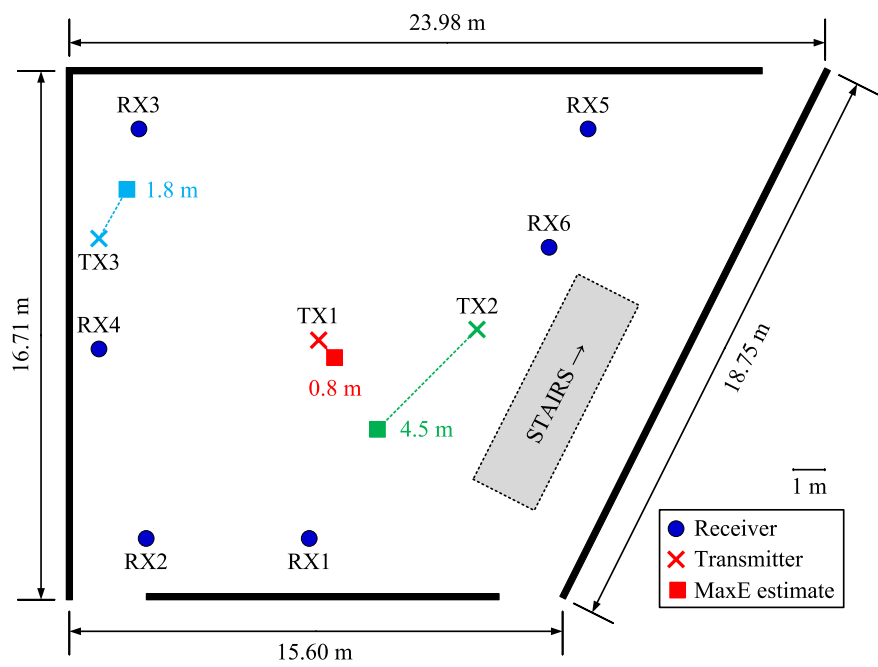

Fig. 6: Localization results with power equalization. Values next to the estimate symbols represent the RMSEs of the location estimates.

more computational complex. The version presented in [8] is not directly applicable to the LWA used in this paper. However, a modified version of SLS is currently under preparation that will be tested and evaluated using the same measurement data as used in this paper.

\section{REFERENCES}

[1] H. Celebi and H. Arslan, "Utilization of location information in cognitive wireless networks," IEEE Wireless Communications, vol. 14, no. 4, pp. 6-13, Aug. 2007.

[2] N. Patwari, J. Ash, S. Kyperountas, A. Hero, R. Moses, and N. Correal, "Locating the nodes: Cooperative localization in wireless sensor networks," IEEE Signal Processing Magazine, vol. 22, no. 4, pp. 54-69, Jul. 2005.

[3] A. Weiss, "On the accuracy of a cellular location system based on RSS measurements," IEEE Transactions on Vehicular Technology, vol. 52, no. 6, pp. 1508-1518, Nov. 2003.

[4] R. Martin and R. Thomas, "Algorithms and bounds for estimating location, directionality, and environmental parameters of primary spectrum users," IEEE Transactions on Wireless Communications, vol. 8, no. 11, pp. $5692-5701$, Nov. 2009.

[5] F. Penna and D. Cabric, "Bounds and tradeoffs for cooperative DoAonly localization of primary users," in Proc. IEEE Global Telecommunications Conference (GLOBECOM), Dec. 2011, pp. 1-5.

[6] J. Wang, J. Chen, and D. Cabric, "Cramer-Rao bounds for joint RSS/DoA-based primary-user localization in cognitive radio networks," IEEE Transactions on Wireless Communications, vol. 12, no. 3, pp. 1363-1375, Mar. 2013.

[7] J. Werner, A. Hakkarainen, and M. Valkama, "Cramer-Rao bound for hybrid RSS-DOA based position and transmit power estimation in cognitive radio systems," in Proc. IEEE 78th Vehicular Technology Conference (VTC Fall), Sep. 2013.

[8] J. Werner, J. Wang, A. Hakkarainen, M. Valkama, and D. Cabric, "Primary user localization in cognitive radio networks using sectorized antennas," in Proc. 10th Annual Conference on Wireless On-demand Network Systems and Services (WONS), Mar. 2013, pp. 155-161.

[9] R. Schmidt, "Multiple emitter location and signal parameter estimation," IEEE Transactions on Antennas and Propagation, vol. 34, no. 3, pp. 276 - 280, Mar. 1986.

[10] J. Werner, J. Wang, A. Hakkarainen, M. Valkama, and D. Cabric, "Primary user DoA and RSS estimation in cognitive radio networks using sectorized antennas," in Proc. 8th International Conference on Cognitive Radio Oriented Wireless Networks (CROWNCOM), Jul. 2013, pp. 43-48. 
[11] H. Paaso, A. Mammela, D. Patron, and K. R. Dandekar, "DoA estimation through modified unitary MUSIC algorithm for CRLH leaky-wave antennas," in Proc. IEEE 24th International Symposium on Personal Indoor and Mobile Radio Communications (PIMRC), Sep. 2013, pp. 311-315.

[12] R. Stansfield, "Statistical theory of d.f. fixing," Journal of the Institution of Electrical Engineers - Part IIIA: Radiocommunication, vol. 94, no. 15, pp. 762-770, 1947.

[13] "Mango communications WARP v3 kit," 2014. [Online]. Available: http://mangocomm.com/products/kits/warp-v3-kit

[14] T. A. Milligan, Modern Antenna Design, 2nd ed. Hoboken, N.J.: IEEE
Press: Wiley-Interscience, 2005.

[15] D. Piazza, D. Michele, and K. Dandekar, "Two port reconfigurable CRLH leaky wave antenna with improved impedance matching and beam tuning," in Proc. 3rd European Conference on Antennas and Propagation (EuCAP), Mar. 2009, pp. 2046-2049.

[16] J. Wang, J. Werner, M. Valkama, and D. Cabric, "Performance analysis of primary user RSS/DoA estimation and localization in cognitive radio networks using sectorized antennas," IEEE Wireless Communications Letters, vol. 3, no. 2, pp. 237-240, Apr. 2014. 